\section{Fórum: saúde e povos indígenas no Brasil. Introdução}

\author{
Forum: health and indigenous peoples in Brazil. \\ Introduction
}

\author{
Foro: salud y pueblos indígenas en Brasil. \\ Introducción
}

\begin{abstract}
This Forum on Health and Indigenous Peoples in Brazil explores contemporary challenges to indigenous health and health politics in Brazil. The short collection of articles that follow are based on presentations, originally given at the Indigenous Health Working Group panel at the 10th Brazilian Public Health Conference in Rio Grande do Sul State, by professors Carlos E. A. Coimbra Jr. (Escola Nacional de Saúde Pública, Fundação Oswaldo Cruz), Marina Denise Cardoso (Universidade Federal de São Carlos) and Eliana E. Diehl (Universidade Federal de Santa Catarina) with Marcos A. Pellegrini (Universidade Federal de Roraima). In this short Introduction, I introduce these contributions, taking as a point of reference a local example of healthcare inequity derived from a presentation at the same panel by Paulo F. Supretaprã, indigenous community leader from Etênhiritipá village, Mato Grosso State.
\end{abstract}

Health of Indigenous Peoples; Indigenous Health Services; Health Policy; Indigenous Population
James R. Welch 1

\section{Resumo}

O presente Fórum sobre Saúde e Povos Indígenas no Brasil explora os desafios contemporâneos para a saúde indígena e políticas de saúde no Brasil. O pequeno conjunto de artigos que se seguem são baseados em palestras, originalmente realizadas em painel do Grupo de Trabalho em Saúde Indígena, por ocasião do 10o Congresso Brasileiro de Saúde Coletiva no Rio Grande do Sul, pelos professores Carlos E. A. Coimbra Jr. (Escola Nacional de Saúde Pública Sergio Arouca, Fundação Oswaldo Cruz), Marina Denise Cardoso (Universidade Federal de São Carlos) $e$ Eliana E. Diehl (Universidade Federal de Santa Catarina), com Marcos A. Pellegrini (Universidade Federal de Roraima). Nesta breve Introdução apresento as contribuições ao Fórum, tomando como ponto de referência um exemplo local de iniquidade nos cuidados à saúde derivado da apresentação realizada no mesmo painel por Paulo F. Supretaprã, liderança comunitária indígena da aldeia Etênhiritipá no Mato Grosso.

Saúde de Populações Indígenas; Serviços de Saúde do Indígena; Política de Saúde; População Indígena 
Durante o 10ํ Congresso Brasileiro de Saúde Coletiva, realizado na Universidade Federal do Rio Grande do Sul (UFRGS), de 14 a 18 de novembro de 2012, o Grupo de Trabalho (GT) Saúde Indígena da Associação Brasileira de Saúde Coletiva (ABRASCO) promoveu o painel Saúde e Povos Indígenas no Brasil: Desafios Nacionais e Insights Locais, por mim coordenado. Participaram do painel Paulo F. Supretaprã, líder comunitário na área de políticas de saúde da aldeia Xavante de Etênhiritipá no Mato Grosso, e os professores Carlos E. A. Coimbra Jr. (Escola Nacional de Saúde Pública Sergio Arouca da Fundação Oswaldo Cruz), Marina Denise Cardoso (Universidade Federal de São Carlos) e Eliana E. Diehl (Universidade Federal de Santa Catarina), esta última acompanhada pelo coautor Marcos A. Pellegrini (Universidade Federal de Roraima). Cada palestrante enfocou uma dimensão específica referente aos complexos desafios contemporâneos do subsistema de atenção à saúde dos povos indígenas no Brasil, sob a responsabilidade da Secretaria Especial de Saúde Indígena (SESAI). Não obstante a diversidade dos temas tratados, as distintas falas foram unificadas pelo foco comum nas marcantes disparidades em saúde que distanciam os povos indígenas do restante da população nacional. Ao final das apresentações, aqui reproduzidas no formato de artigos ou ensaios, fica evidente que, apesar de os princípios da equidade e da atenção diferenciada serem norteadores do Subsistema de Atenção à Saúde Indígena (SASISUS), a realidade da saúde dos povos indígenas no Brasil continua distante do ideal.

O primeiro palestrante, Supretaprã, listou vários dos desafios enfrentados pelos serviços de saúde destinados aos povos indígenas, tomando como exemplo o Distrito Sanitário Especial Indígena (DSEI) Xavante no Mato Grosso. Supreteprã, ex-cacique da aldeia Etênhiritipá, atua como líder articulador das aldeias da Terra Indígena (TI) Pimentel Barbosa em prol de melhorias nos serviços de saúde oferecidos às comunidades dessa TI. Em sua fala, Supreteprã chamou atenção para as circunstâncias que limitam o acesso das aldeias aos serviços de saúde, assim como para as precárias estatísticas de saúde apresentadas pela população da TI, em comparação às estatísticas regionais e nacionais. Apesar de, em vários aspectos, as circunstâncias relatadas por Supreteprã serem particulares ao contexto no qual estão inseridas, as principais deficiências no sistema de saúde por ele identificadas para o DSEI Xavante foram também identificadas pelos demais painelistas, a ponto de serem quase universais, independentemente do DSEI ao qual determinada TI ou comunidade esteja jurisdicionada.
Segundo Supretaprã, a população Xavante totaliza cerca de 17 mil pessoas e reside em cerca de 220 aldeias situadas em dez TI descontínuas em Mato Grosso. A atenção à saúde da totalidade da população está a cargo do DSEI Xavante, sediado no extremo sul da área Xavante, na cidade de Barra do Garças. Apesar de a maioria da população Xavante estar situada relativamente próxima à sede do DSEI, onde, aliás, também está localizada a única Casa de Saúde do Índio (CASAI) de toda a região, as aldeias situadas nas TI do norte, incluindo Pimentel Barbosa, estão muito distantes de Barra do Garças, não raro superando $400 \mathrm{~km}$. Além disso, a aldeia de Supreteprã, Etênhiritipá, assim como as demais aldeias da TI Pimentel Barbosa, distam pelo menos $150 \mathrm{~km}$ do polo-base de referência, na cidade de Água Boa. As grandes distâncias a serem vencidas para a realização de exames ou de quaisquer procedimentos, mesmo os mais simples, agravadas pela deficiente comunicação entre as aldeias e a sede do polo-base (não há rádios e os telefones do tipo "orelhão" existentes em algumas aldeias frequentemente apresentam defeitos) constituem motivos de queixas constantes por parte dos indígenas e limitam o acesso destes aos serviços de saúde.

Ainda de acordo com Supretaprã, o DSEI Xavante falha por não assegurar de maneira ininterrupta os serviços mais rotineiros previstos na atenção básica, assim como o tratamento de doentes crônicos (constantemente faltam insulina ou anti-hipertensivos no posto de saúde, apesar de várias pessoas na aldeia precisarem desta medicação diariamente). As equipes de saúde visitam as aldeias da TI Pimentel Barbosa de maneira irregular e sem avisar, o que retarda a realização de diagnósticos, dificulta o acompanhamento de pacientes em tratamento e atrapalha o calendário vacinal. A infraestrutura dos poucos postos de saúde existentes na TI Pimentel Barbosa também é precária: os postos não oferecem conforto ou privacidade para o atendimento adequado aos pacientes, não existe habitação adequada para os profissionais de saúde que residem nas aldeias e não há manejo seguro do lixo; este é lançado em um buraco raso e deixado a céu aberto, expondo crianças e animais ao contato com materiais contaminados. Supretaprã também chamou atenção para a irregularidade do acompanhamento das gestantes e do atendimento odontológico nas aldeias, o limitado acesso à água potável de qualidade e a tênue atuação do DSEI na intermediação dos serviços de saúde municipais quando, não raro, pacientes indígenas ficam sozinhos em enfermarias coletivas, sem informação e em condições precárias.

As deficiências dos serviços de saúde relatados por Supretaprã com respeito à sua própria 
aldeia, terra indígena e etnia, ilustram com base no caso de um DSEI no Mato Grosso, os desafios sistêmicos e problemas enfrentados na implementação da Política Nacional de Atenção à Saúde dos Povos Indígenas (PNASPI). A população indígena no Brasil é muito diversificada, assim como também são os contextos regionais nos quais se inserem os 34 DSEIs existentes no país. Apesar de o cenário descrito por Supretaprã ser particular, os principais problemas por ele apontados parecem repetir em outras partes do país e em outros contextos, como nos foi possível apreender a partir das demais apresentações.

Indicadores de saúde regionais e nacionais que revelam iniquidades na saúde indígena foi o tópico de outra apresentação durante o painel, e aqui apresentada como o primeiro artigo do Fórum. Nessa, Coimbra Jr. traça um panorama nacional da saúde indígena, com foco nas crianças e mulheres, a partir dos primeiros resultados já publicados do I Inquérito Nacional de Saúde e Nutrição Indígena, realizado pela ABRASCO e uma grande rede de pesquisadores das diferentes regiões do país, com o financiamento da Fundação Nacional de Saúde (FUNASA) e do Banco Mundial. Segundo esse estudo, os povos indígenas no Brasil apresentam recorrentes disparidades em saúde em comparação com a população brasileira não indígena. Além disso, particularidades regionais contribuem para a sua maior vulnerabilidade, com a Região Norte exibindo os mais divergentes indicadores de saúde em comparação ao restante do país. Coimbra Jr. enfatiza que as disparidades nos perfis de saúde indígena observados nos níveis nacional e regional resultam de complexa combinação de fatores socioeconômicos, políticos e ambientais que precisam ser melhor entendidos. Dentre as principais contribuições desse inquérito, que pode ser considerado como um marco na saúde coletiva brasileira, podemos destacar a documentação sistemática de vários indicadores de saúde que exprimem algumas das principais vulnerabilidades às quais esses povos estão sujeitos. As análises resultantes desse banco de dados permitirão identificar prioridades da saúde indígena segundo as macrorregiões do país, levando em consideração o papel dinâmico da crescente participação no mercado e da globalização entre os povos indígenas.

No segundo artigo, Cardoso destaca que os DSEI foram concebidos segundo o princípio da distritalização dos serviços, de modo a melhor atender às necessidades específicas das diferentes etnias indígenas. Mais uma vez, surge o tema da diversidade sociocultural como elemento fundante da política de saúde para os povos indígenas no Brasil. A vulnerabilidade dos povos indígenas e seus desafios e necessidades particulares estão na raiz dos princípios de "atenção diferenciada" e "controle social", segundo os quais as especificidades das comunidades requerem serviços igualmente específicos e concebidos de maneira apropriada às particularidades de cada contexto. Cardoso explora esses princípios dispensando particular atenção às suas histórias na arena político-burocrática dos cuidados à saúde no Brasil. As limitações por ela identificadas fazem coro com as reivindicações de Supretaprã por um DSEI cuja atuação seja sensível às necessidades de uma população diversa e geograficamente dispersa. Seu apelo por melhoras nos serviços de saúde não deve ser visto como uma demanda por direitos especiais, mas sim como a reiteração dos princípios de um Sistema Único de Saúde (SUS) realmente inclusivo.

O terceiro artigo, de Diehl \& Pellegrini, enfoca outra deficiência do SASISUS destacada na fala de Supretaprã - a formação e educação permanente de profissionais de saúde. Enquanto Supretaprã chamou atenção para as lacunas no treinamento e educação profissional continuada para profissionais de saúde tanto indígenas quanto não indígenas no DSEI Xavante, Diehl \& Pellegrini enfocaram a questão do (des)preparo dos profissionais não indígenas para atuarem no âmbito de comunidades diversas do ponto de vista social e linguístico. Os autores propõem que a caracterização de sociedades indígenas como vulneráveis não justifica a aplicação uniforme de intervenções biomédicas sem consideração para as diferenças étnicas, em nome de uma "salvação médica". Em consonância com alguns pontos semelhantes feitos por Supretaprã, Coimbra Jr. e Cardoso acerca da importância de serviços diferenciados regionalmente, Diehl \& Pellegrini frisam que os profissionais lotados nos vários DSEIs requerem capacitação adequada para que possam melhor atender às necessidades das comunidades indígenas sob suas jurisdições.

Os artigos que compõem esse Fórum carregam consigo forte mensagem acerca das deficiências atuais e enormes desafios futuros para a implementação plena de uma política de atenção diferenciada à saúde dos povos indígenas no Brasil. Convido o leitor a ler cada um dos artigos que fazem parte desse Fórum, tendo em mente os exemplos trazidos por Supretaprã, baseados em sua vivência na TI Pimentel Barbosa. Sua mensagem certamente se aplica ao contexto vivenciado por outras etnias nas diferentes regiões do país, e encapsula os múltiplos desafios a serem enfrentados para que, efetivamente, os direitos dos povos indígenas a uma saúde pública plena e de qualidade sejam atingidos. 


\section{Resumen}

El presente Foro sobre salud y pueblos indígenas en Brasil explora los desafíos contemporáneos para la salud indígena y las políticas de salud en Brasil. El pequeño conjunto de artículos que siguen se basan en conferencias, originalmente formuladas en el panel del Grupo de Trabajo sobre Salud Indígena, realizado durante el 10 o Congreso Brasileño de Salud Colectiva, en Río Grande do Sul, por los profesores Carlos E. A. Coimbra Jr. (Escuela Nacional de Salud Pública, Fundación Oswaldo Cruz), Marina Denise Cardoso (Universidad Federal de São Carlos) y Eliana E. Diehl (Universidad Federal de Santa Catarina), con Marcos A. Pellegrini (Universidad
Federal de Roraima). En esta breve introducción, les presento las contribuciones al foro, tomando como referencia un ejemplo local de iniquidad en la atención de salud, derivado de la ponencia realizada en el mismo panel por Paulo F. Supretaprã, líder comunitario de la aldea Etênhiritipá en el Estado de Mato Grosso.

Salud de Poblaciones Indígenas; Servicios de Salud del Indígena; Política de Salud; Población Indígena

Recebido em 26/Fev/2014

Aprovado em 12/Mar/2014 